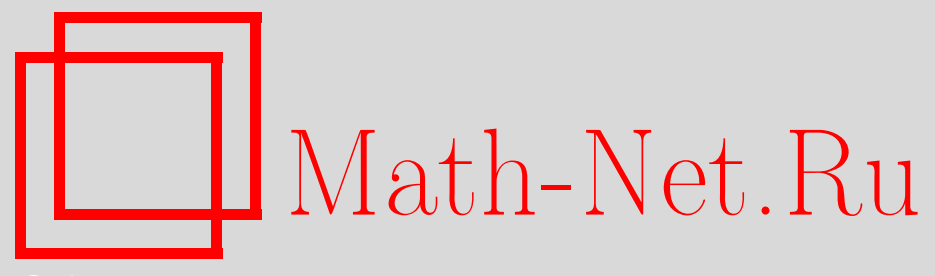

T. К. Юлдашев, Обратная задача для нелинейного интегро-дифференциального уравнения Фредгольма четвертого порядка с вырожденным ядром, Вестн. Сам. гос. техн. ун-та. Сер. Физ.-мат. науки, 2015, номер 4, 736-749

DOI: https://doi.org/10.14498/vsgtu1434

Использование Общероссийского математического портала MathNet.Ru подразумевает, что вы прочитали и согласны с пользовательским соглашением

http: //www . mathnet.ru/rus/agreement

Параметры загрузки:

IP: 18.234 .197 .8

26 апреля 2023 г., 10:23:23

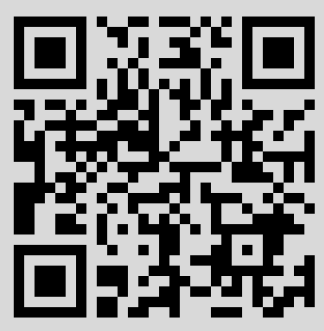


УДК 517.968.21

\title{
ОБРАТНАЯ ЗАДАЧА ДЛЯ НЕЛИНЕЙНОГО ИНТЕГРО-ДИФФЕРЕНЦИАЛЬНОГО УРАВНЕНИЯ ФРЕДГОЛЬМА ЧЕТВЕРТОГО ПОРЯДКА С ВЫРОЖДЕННЫМ ЯДРОМ
}

\section{T. К. Юлдашев}

Сибирский государственный аэрокосмический университет им. ак. М. Ф. Решетнева, Россия, 660014, Красноярск, пр. имени газеты «Красноярский рабочий», 31.

\begin{abstract}
Аннотация
Рассмотрены вопросы об однозначной разрешимости обратной задачи для одного нелинейного интегро-дифференциального уравнения типа Фредгольма в частных производных четвертого порядка с вырожденным ядром. Развит метод вырожденного ядра в случае обратной задачи для рассматриваемого интегро-дифференциального уравнения типа Фредгольма в частных производных четвертого порядка. С помощью обозначения интегро-дифференциальное уравнение типа Фредгольма сведено к системе интегральных уравнений. Система интегральных уравнений путем дифференцирования сведена к системе дифференциальных уравнений. При выполнении определенного условия система дифференциальных уравнений заменена системой алгебраических уравнений. При регулярных значениях спектрального параметра решена система алгебраических уравнений методом Крамера. Использование дополнительного условия относительно основной неизвестной функции позволило получить нелинейное интегральное уравнение типа Вольтерра второго рода и относительно функции восстановления получить специальное интегральное уравнение типа Вольтерра первого рода. Применен метод последовательных приближений в сочетании с методом сжимающих отображений. Далее определяется функция восстановления. Данная работа является дальнейшим развитием теории интегро-дифференциальных уравнений типа Фредгольма с вырожденным ядром.
\end{abstract}

Ключевые слова: обратная задача, интегро-дифференциальное уравнение, уравнение типа Фредгольма, вырожденное ядро, система интегральных уравнений, однозначная разрешимость.

doi: http://dx.doi.org/10.14498/vsgtu1434

Постановка задачи. Математическое моделирование многих процессов, происходящих в реальном мире, часто приводит к необходимости изучения прямых и обратных задач для уравнений неклассической математической физики. С точки зрения физических приложений представляют большой интерес

(C) 2015 Самарский государственный технический университет.

\section{Образец для цитирования}

Юлдашев Т. К. Обратная задача для нелинейного интегро-дифференциального уравнения Фредгольма четвертого порядка с вырожденным ядром // Becmн. Cам. гос. техн. ун-та. Сер. Физ.-мат. науки, 2015. Т. 19, № 4. С. 736-749. doi: 10.14498/vsgtu1434.

Сведения об авторе

Турсун Камалдинович Юлдашев (к.ф.-м.н., доц.; tursunbay@rambler.ru), доцент, каф. высшей математики. 
дифференциальные уравнения в частных производных высоких порядков. Многие задачи газовой динамики, теории упругости, теории пластин и оболочек приводят к рассмотрению дифференциальных уравнений в частных производных высоких порядков [1]. Изучению уравнений в частных производных четвертого порядка посвящено большое количество работ (см., например, [2-10]).

Теория обратных задач представляет собой активно развивающееся направление современной теории дифференциальных уравнений. Интенсивное исследование обратных задач обусловлено и необходимостью разработки математических методов решения прикладных проблем. Обратную задачу называют линейной, если функция восстановления входит в данное уравнение линейно. Линейные обратные задачи рассматривались, в частности, в [11-20]. Нелинейные обратные задачи рассматривались в работах [21-25].

В настоящей работе предлагается методика изучения обратной задачи для нелинейного интегро-дифференциального уравнения типа Фредгольма в частных производных четвертого порядка с вырожденным ядром. Она является дальнейшим продолжением и совершенствованием методики работ [26-29].

Итак, в области $\Omega \equiv \Omega_{T} \times \Omega_{l}, \Omega_{T} \equiv[0, T], \Omega_{l} \equiv[0, l]$, рассматривается интегро-дифференциальное уравнение типа Фредгольма с вырожденным ядром:

$$
\begin{gathered}
\frac{\partial^{4} u(t, x)}{\partial t^{2} \partial x^{2}}+\lambda \int_{0}^{T} K(t, s) \frac{\partial^{2} u(s, x)}{\partial s \partial x} d s= \\
=p(t) \beta(x)+f\left(x, \int_{0}^{T} \int_{0}^{l} H(s, y) u(s, y) d y d s\right)
\end{gathered}
$$

с условиями

$$
\begin{gathered}
u(0, x)=\varphi_{1}(x), \quad u_{t}(0, x)=\varphi_{2}(x), \quad x \in \Omega_{l}, \\
u(t, 0)=\psi_{1}(t), \quad u_{t x}(t, 0)=\psi_{2}(t), \quad t \in \Omega_{T}, \\
u\left(t_{0}, x\right)=\eta(x), \quad 0<t_{0}<T, \quad x \in \Omega_{l},
\end{gathered}
$$

где

$$
\begin{gathered}
p(t) \in C\left(\Omega_{T}\right), \quad f(x, \gamma) \in C\left(\Omega_{l} \times \mathbb{R}\right) ; \\
\varphi_{k}(x) \in C^{2}\left(\Omega_{l}\right), \quad \psi_{k}(t) \in C^{2}\left(\Omega_{T}\right), \quad k=1,2 ; \\
\psi_{1}(0)=\varphi_{1}(0), \quad \psi_{1}^{\prime}(0)=\varphi_{2}(0), \quad \psi_{2}^{\prime}(0)=\varphi_{2}^{\prime}(0) ; \\
K(t, s)=\sum_{i=1}^{n} a_{i}(t) b_{i}(s), \quad 0<a_{i}(t), b_{i}(s) \in C\left(\Omega_{T}\right) ;
\end{gathered}
$$

$\eta(x) \in C^{2}\left(\Omega_{l}\right) ; \beta(x) \in C\left(\Omega_{l}\right)-$ функция восстановления; $\lambda$ - параметр;

$$
0<\int_{0}^{T} \int_{0}^{l}|H(t, x)| d x d t<\infty .
$$

Под решением обратной задачи (1)-(4) будем понимать пару функций $\left\{u(t, x) \in C^{2,2}(\Omega), \beta(x) \in C\left(\Omega_{l}\right)\right\}$, удовлетворяющую уравнению (1) и условиям $(2)-(4)$. 
1. Начальная задача (1)-(3). В уравнении (1) сделаем замену $u_{t x}(t, x)=$ $=\vartheta(t, x)$. Тогда оно приобретает вид

$$
\begin{aligned}
& \frac{\partial^{2} \vartheta(t, x)}{\partial t \partial x}+\lambda \int_{0}^{T} K(t, s) \vartheta(s, x) d s= \\
& \quad=p(t) \beta(x)+f\left(x, \int_{0}^{T} \int_{0}^{l} H(s, y) u(s, y) d y d s\right) .
\end{aligned}
$$

С помощью обозначения

$$
c_{i}(x)=\int_{0}^{T} b_{i}(s) \vartheta(s, x) d s
$$

уравнение (5) перепишется в следующем виде:

$$
\frac{\partial^{2} \vartheta(t, x)}{\partial t \partial x}=-\lambda \sum_{i=1}^{n} a_{i}(t) c_{i}(x)+p(t) \beta(x)+f\left(x, \int_{0}^{T} \int_{0}^{l} H(s, y) u(s, y) d y d s\right) .
$$

В силу постановки задачи правая часть этого выражения суть непрерывные функции своих аргументов. Путем интегрирования по $x$ и по $t$ из последнего равенства получаем

$$
\begin{aligned}
\vartheta(t, x)=D_{1} & (x)+\int_{0}^{t} E_{1}(s) d s-\lambda \sum_{i=1}^{n} \int_{0}^{t} a_{i}(s) d s \int_{0}^{x} c_{i}(y) d y+ \\
& +q(t) \int_{0}^{x} \beta(y) d y+t \int_{0}^{x} f\left(y, \int_{0}^{T} \int_{0}^{l} H(\theta, z) u(\theta, z) d z d \theta\right) d y,
\end{aligned}
$$

где $D_{1}(x) \in C^{2}\left(\Omega_{l}\right), E_{1}(t) \in C^{2}\left(\Omega_{T}\right)$ - произвольные функции, которые подлежат определению,

$$
q(t)=\int_{0}^{t} p(s) d s
$$

Подставляя (7) в (6), имеем

$$
\begin{aligned}
c_{i}(x)= & \int_{0}^{T} b_{i}(s)\left[D_{1}(x)+\int_{0}^{s} E_{1}(\theta) d \theta-\lambda \sum_{i=1}^{n} \int_{0}^{s} a_{i}(\theta) d \theta \int_{0}^{x} c_{i}(y) d y+\right. \\
& \left.+q(s) \int_{0}^{x} \beta(y) d y+s \int_{0}^{x} f\left(y, \int_{0}^{T} \int_{0}^{l} H(\theta, z) u(\theta, z) d z d \theta\right) d y\right] d s .
\end{aligned}
$$

Обозначим

$$
\begin{aligned}
B_{i}(x)= & \int_{0}^{T} b_{i}(s)\left[D_{1}(x)+\int_{0}^{s} E_{1}(\theta) d \theta+\right. \\
& \left.+q(s) \int_{0}^{x} \beta(y) d y+s \int_{0}^{x} f\left(y, \int_{0}^{T} \int_{0}^{l} H(\theta, z) u(\theta, z) d z d \theta\right) d y\right] d s .
\end{aligned}
$$


Пусть

$$
A_{i j}=\int_{0}^{T} b_{i}(s) \int_{0}^{s} a_{i}(\theta) d \theta d s>0 .
$$

Тогда (8) запишем в виде следующей системы интегральных уравнений (СИУ):

$$
c_{i}(x)+\lambda \sum_{j=1}^{n} A_{i j} \int_{0}^{x} c_{j}(y) d y=B_{i}(x), \quad i=\overline{1, n} .
$$

Функции, входящие в СИУ (11), являются непрерывно дифференцируемыми по $x$. Поэтому, дифференцируя систему (11) получим

$$
c_{i}^{\prime}(x)+\lambda \sum_{j=1}^{n} A_{i j} c_{j}(x)=B_{i}^{\prime}(x), \quad i=\overline{1, n} .
$$

Вместо СИУ (11) рассмотрим систему дифференциальных уравнений (СДУ) (12). Будем решать СДУ (12) при выполнении следующего условия:

$$
c_{i}^{\prime}(x)=-\omega c_{i}(x), \quad 0<\omega=\text { const }, \quad i=\overline{1, n} .
$$

Если условие (13) выполняется, то из СДУ (12), получим систему алгебраических уравнений (САУ)

$$
c_{i}(x)-\frac{\lambda}{\omega} \sum_{j=1}^{n} A_{i j} c_{j}(x)=-\frac{1}{\omega} B_{i}^{\prime}(x), i=\overline{1, n} .
$$

САУ (14) однозначно разрешима при любых конечных $B_{i}^{\prime}(x)$, если выполняется условие

$$
\Delta(\lambda)=\left|\begin{array}{cccc}
1-\frac{\lambda}{\omega} A_{11} & -\frac{\lambda}{\omega} A_{12} & \ldots & -\frac{\lambda}{\omega} A_{1 n} \\
-\frac{\lambda}{\omega} A_{21} & 1-\frac{\lambda}{\omega} A_{22} & \ldots & -\frac{\lambda}{\omega} A_{2 n} \\
\vdots & \vdots & \ddots & \vdots \\
-\frac{\lambda}{\omega} A_{n 1} & -\frac{\lambda}{\omega} A_{n 2} & \ldots & 1-\frac{\lambda}{\omega} A_{n n}
\end{array}\right| \neq 0
$$

Определитель $\Delta(\lambda)$ в $(15)$ есть многочлен относительно $\lambda$ степени не выше $n$. Уравнение $\Delta(\lambda)=0$ имеет не более $n$ различных корней. Эти корни являются собственными числами ядра интегро-дифференциального уравнения (1). Другие значения $\lambda$ являются регулярными, при которых условие (15) выполняется. Для регулярных значений $\lambda$ система (14) имеет единственное решение при любой конечной правой части. В настоящей работе для таких регулярных значений параметра $\lambda$ устанавливается однозначная разрешимость поставленной обратной задачи (1)-(4).

Решение системы алгебраических уравнений (14) запишем в виде

$$
c_{i}(x)=\frac{\Delta_{i}(\lambda, x)}{\Delta(\lambda)}, \quad i=\overline{1, n},
$$


где

$$
\Delta_{i}(\lambda, x)=\left|\begin{array}{ccccccc}
1-\frac{\lambda}{\omega} A_{11} & \ldots & -\frac{\lambda}{\omega} A_{1(i-1)} & -\frac{1}{\omega} B_{1}(x) & -\frac{\lambda}{\omega} A_{1(i+1)} & \ldots & -\frac{\lambda}{\omega} A_{1 n} \\
-\frac{\lambda}{\omega} A_{21} & \ldots & -\frac{\lambda}{\omega} A_{2(i-1)} & -\frac{1}{\omega} B_{2}(x) & -\frac{\lambda}{\omega} A_{2(i+1)} & \ldots & -\frac{\lambda}{\omega} A_{2 n} \\
\vdots & \vdots & \vdots & \vdots & \vdots & \ddots & \vdots \\
-\frac{\lambda}{\omega} A_{n 1} & \ldots & -\frac{\lambda}{\omega} A_{n(i-1)} & -\frac{1}{\omega} B_{n}(x) & -\frac{\lambda}{\omega} A_{n(i+1)} & \ldots & 1-\frac{\lambda}{\omega} A_{n n}
\end{array}\right| .
$$

Среди элементов определителей $\Delta_{i}(\lambda, x)$ находятся функции $B_{i}^{\prime}(x)$. В свою очередь, в составе функций $B_{i}^{\prime}(x)$ находятся пока неизвестные функции $D_{1}^{\prime}(x)$, $u(t, x)$ и $\beta(x)$. В самом деле, эти неизвестные функции находились в правой части САУ (14). Чтобы вывести их из знака определителей, продифференцируем (9), а полученное выражение запишем в следующем виде:

$$
B_{i}^{\prime}(x)=D_{1}^{\prime}(x) B_{1 i}+f\left(x, \int_{0}^{T} \int_{0}^{l} H(\theta, y) u(\theta, y) d y d \theta\right) B_{2 i}+\beta(x) B_{3 i},
$$

где

$$
B_{1 i}=\int_{0}^{T} b_{i}(s) d s, \quad B_{2 i}=\int_{0}^{T} s b_{i}(s) d s, \quad B_{3 i}=\int_{0}^{T} q(s) b_{i}(s) d s .
$$

В этом случае, согласно свойству определителей имеем

$\Delta_{i}(\lambda, x)=D_{1}^{\prime}(x) \Delta_{1 i}(\lambda)+f\left(x, \int_{0}^{T} \int_{0}^{l} H(\theta, y) u(\theta, y) d y d \theta\right) \Delta_{2 i}(\lambda)+\beta(x) \Delta_{3 i}(\lambda)$, где

$$
\Delta_{k i}(\lambda)=\left|\begin{array}{ccccccc}
1-\frac{\lambda}{\omega} A_{11} & \ldots & -\frac{\lambda}{\omega} A_{1(i-1)} & -\frac{1}{\omega} B_{k 1} & -\frac{\lambda}{\omega} A_{1(i+1)} & \ldots & -\frac{\lambda}{\omega} A_{1 n} \\
-\frac{\lambda}{\omega} A_{21} & \ldots & -\frac{\lambda}{\omega} A_{2(i-1)} & -\frac{1}{\omega} B_{k 2} & -\frac{\lambda}{\omega} A_{2(i+1)} & \ldots & -\frac{\lambda}{\omega} A_{2 n} \\
\vdots & \vdots & \vdots & \vdots & \vdots & \ddots & \vdots \\
-\frac{\lambda}{\omega} A_{n 1} & \ldots & -\frac{\lambda}{\omega} A_{n(i-1)} & -\frac{1}{\omega} B_{k n} & -\frac{\lambda}{\omega} A_{n(i+1)} & \ldots & 1-\frac{\lambda}{\omega} A_{n n}
\end{array}\right|,
$$

$k=\overline{1,3}$.

Тогда формула (16) переписывается в виде

$$
\begin{aligned}
c_{i}(x) & =D_{1}^{\prime}(x) \frac{\Delta_{1 i}(\lambda)}{\Delta(\lambda)}+ \\
& +f\left(x, \int_{0}^{T} \int_{0}^{l} H(\theta, y) u(\theta, y) d y d \theta\right) \frac{\Delta_{2 i}(\lambda)}{\Delta(\lambda)}+\beta(x) \frac{\Delta_{3 i}(\lambda)}{\Delta(\lambda)}, \quad i=\overline{1, n} .
\end{aligned}
$$

Итак, решена САУ (14):

$$
\begin{aligned}
c_{i}(x) & -\frac{\lambda}{\omega} \sum_{j=1}^{n} A_{i j} c_{j}(x)= \\
& =-\frac{1}{\omega}\left[D_{1}^{\prime}(x) B_{1 i}+f\left(x, \int_{0}^{T} \int_{0}^{l} H(\theta, y) u(\theta, y) d y d \theta\right) B_{2 i}+\beta(x) B_{3 i}\right],
\end{aligned}
$$


$i=\overline{1, n}$, а решения представлены в виде функций $(17)$.

Чтобы получить решение СДУ (12), функции (17) подставим в условие (13):

$$
\begin{aligned}
& c_{i}^{\prime}(x)=-\omega\left[D_{1}^{\prime}(x) \frac{\Delta_{1 i}(\lambda)}{\Delta(\lambda)}+\right. \\
& \left.\quad+f\left(x, \int_{0}^{T} \int_{0}^{l} H(\theta, y) u(\theta, y) d y d \theta\right) \frac{\Delta_{2 i}(\lambda)}{\Delta(\lambda)}+\beta(x) \frac{\Delta_{3 i}(\lambda)}{\Delta(\lambda)}\right], \quad i=\overline{1, n} .
\end{aligned}
$$

В (6) учтем второе условие из (3). Тогда из (6) получим следующее начальное условие для интегрирования функций (18):

$$
c_{i}(0)=\int_{0}^{T} b_{i}(s) \psi_{2}(s) d s, \quad i=\overline{1, n} .
$$

Из (18) интегрированием получаем решение системы неоднородного дифференциального уравнения (12):

$$
\begin{gathered}
c_{i}(x)=\int_{0}^{T} b_{i}(s) \psi_{2}(s) d s-\omega\left[D_{1}(x) \frac{\Delta_{1 i}(\lambda)}{\Delta(\lambda)}+\right. \\
+\int_{0}^{x} f\left(y, \int_{0}^{T} \int_{0}^{l} H(\theta, z) u(\theta, z) d z d \theta\right) d y \frac{\Delta_{2 i}(\lambda)}{\Delta(\lambda)}+ \\
\left.\quad+\int_{0}^{x} \beta(y) d y \frac{\Delta_{3 i}(\lambda)}{\Delta(\lambda)}\right], i=\overline{1, n} .
\end{gathered}
$$

Подстановка (19) в (7) дает следующее выражение:

$$
\begin{aligned}
& u_{t x}(t, x)=D_{1}(x)+\int_{0}^{t} E_{1}(s) d s+ \\
& +\lambda \sum_{i=1}^{n} \int_{0}^{t} a_{i}(s) d s \int_{0}^{x}\left\{-\int_{0}^{T} b_{i}(s) \psi_{2}(s) d s+\right. \\
& +\omega D_{1}(y) \frac{\Delta_{1 i}(\lambda)}{\Delta(\lambda)}+\omega \int_{0}^{y} f\left(z, \int_{0}^{T} \int_{0}^{l} H(\theta, \xi) u(\theta, \xi) d \xi d \theta\right) \frac{\Delta_{2 i}(\lambda)}{\Delta(\lambda)}+ \\
& \left.\quad+\omega \int_{0}^{y} \beta(z) d z \frac{\Delta_{3 i}(\lambda)}{\Delta(\lambda)}\right\} d y+ \\
& +q(t) \int_{0}^{x} \beta(y) d y+t \int_{0}^{x} f\left(y, \int_{0}^{T} \int_{0}^{l} H(\theta, z) u(\theta, z) d z d \theta\right) d y .
\end{aligned}
$$

Интегрированием по $x$ и по $t$ из (20) получаем

$$
\begin{aligned}
u(t, x)= & D_{2}(x)+\int_{0}^{t} E_{2}(s) d s+t \int_{0}^{x} D_{1}(y) d y+x \int_{0}^{t}(t-s) E_{1}(s) d s+ \\
& +\lambda \sum_{i=1}^{n} \int_{0}^{t}(t-s) a_{i}(s) d s \int_{0}^{x}(x-y)\left\{-\int_{0}^{T} b_{i}(s) \psi_{2}(s) d s+\right.
\end{aligned}
$$




$$
\begin{gathered}
+\omega D_{1}(y) \frac{\Delta_{1 i}(\lambda)}{\Delta(\lambda)}+\omega \int_{0}^{y} f\left(z, \int_{0}^{T} \int_{0}^{l} H(\theta, \xi) u(\theta, \xi) d \xi d \theta\right) \frac{\Delta_{2 i}(\lambda)}{\Delta(\lambda)}+ \\
\left.+\omega \int_{0}^{y} \beta(z) d z \frac{\Delta_{3 i}(\lambda)}{\Delta(\lambda)}\right\} d y+\bar{q}(t) \int_{0}^{x}(x-y) \beta(y) d y+ \\
+\frac{t^{2}}{2} \int_{0}^{x}(x-y) f\left(y, \int_{0}^{T} \int_{0}^{l} H(\theta, z) u(\theta, z) d z d \theta\right) d y
\end{gathered}
$$

где $D_{2}(x) \in C^{2}\left(\Omega_{l}\right), E_{2}(t) \in C^{2}\left(\Omega_{T}\right)$ - произвольные функции, которые подлежат определению;

$$
D_{2}(0)=E_{2}(0)=0, \quad \bar{q}(t)=\int_{0}^{t} q(s) d s .
$$

Используя условия (2) и (3), из (21) приходим к следующим равенствам:

$$
\begin{gathered}
\varphi_{1}(x)=D_{2}(x), \quad \psi_{1}(t)=\int_{0}^{t} E_{2}(s) d s, \\
\varphi_{2}(x)=\int_{0}^{x} D_{1}(y) d y, \quad \psi_{2}(t)=\int_{0}^{t} E_{1}(s) d s .
\end{gathered}
$$

Тогда интегральное уравнение (21) запишется в виде

$$
\begin{aligned}
u(t, x)=Q(t, x)+\int_{0}^{x} F_{1}(t, x, y)\left[\beta(y)+\int_{0}^{y} \beta(z) d z\right] d y+ & \\
+\int_{0}^{x} F_{2}(t, x, y) & {\left[f\left(y, \int_{0}^{T} \int_{0}^{l} H(\theta, z) u(\theta, z) d z d \theta\right)+\right.} \\
+ & \left.\int_{0}^{y} f\left(z, \int_{0}^{T} \int_{0}^{l} H(\theta, \xi) u(\theta, \xi) d \xi d \theta\right) d z\right] d y
\end{aligned}
$$

где

$$
\begin{gathered}
Q(t, x)=\varphi_{1}(x)+\psi_{1}(t)+t \varphi_{2}(x)+x \int_{0}^{t} \psi_{2}(s) d s- \\
-\lambda \sum_{i=1}^{n} \int_{0}^{t}(t-s) a_{i}(s) d s \int_{0}^{x}(x-y)\left[\int_{0}^{T} b_{i}(s) \psi_{2}(s) d s-\omega \varphi_{2}^{\prime}(y) \frac{\Delta_{1 i}(\lambda)}{\Delta(\lambda)}\right] d y \\
F_{1}(t, x, y)=\mu(t)(x-y), \quad F_{2}(t, x, y)=\nu(t)(x-y) \\
\mu(t)=\bar{q}(t)+\omega \lambda \sum_{i=1}^{n} \int_{0}^{t}(t-s) a_{i}(s) d s \frac{\Delta_{3 i}(\lambda)}{\Delta(\lambda)} \\
\nu(t)=\frac{t^{2}}{2}+\omega \lambda \sum_{i=1}^{n} \int_{0}^{t}(t-s) a_{i}(s) d s \frac{\Delta_{2 i}(\lambda)}{\Delta(\lambda)}
\end{gathered}
$$


Итак, начальная задача (1)-(3) свелась к интегральному уравнению (22). Уравнение (22) относительно основной неизвестной функции $u(t, x)$ является интегральным уравнением типа Вольтерра второго рода, а относительно функции восстановления $\beta(x)$ является интегральным уравнением типа Вольтерра первого рода.

2. Теорема об однозначной разрешимости обратной задачи. Учет дополнительного условия (4) в (22) дает

$$
\begin{aligned}
\int_{0}^{x} F_{1}\left(t_{0}, x, y\right) & {\left[\beta(y)+\int_{0}^{y} \beta(z) d z\right] d y+} \\
+ & \int_{0}^{x} F_{2}\left(t_{0}, x, y\right)\left[f\left(y, \int_{0}^{T} \int_{0}^{l} H(\theta, z) u(\theta, z) d z d \theta\right)+\right. \\
& \left.+\int_{0}^{y} f\left(z, \int_{0}^{T} \int_{0}^{l} H(\theta, \xi) u(\theta, \xi) d \xi d \theta\right) d z\right] d y=g(x),
\end{aligned}
$$

где $g(x)=\eta(x)-Q\left(t_{0}, x\right)$.

Двукратным дифференцированием из (23) для функции восстановления $\beta(x)$ получаем следующее соотношение:

$$
\begin{aligned}
\beta(x)+\int_{0}^{x} \beta(y) d y=\bar{g}(x)- & \bar{F}\left(t_{0}\right)\left[f\left(x, \int_{0}^{T} \int_{0}^{l} H(\theta, z) u(\theta, z) d z d \theta\right)+\right. \\
& \left.+\int_{0}^{x} f\left(y, \int_{0}^{T} \int_{0}^{l} H(\theta, z) u(\theta, z) d z d \theta\right) d y\right],
\end{aligned}
$$

где $\bar{g}(x)=g^{\prime \prime}(x) / \mu\left(t_{0}\right), \bar{F}\left(t_{0}\right)=\nu\left(t_{0}\right) / \mu\left(t_{0}\right)$.

Подставляя (24) в $(22)$, относительно основной неизвестной функции $u(t, x)$ окончательно получим следующее интегральное уравнение типа Вольтерра второго рода:

$$
\begin{aligned}
u(t, x)=h(t, x)+\int_{0}^{x} \Phi(t, & x, y)\left[f\left(y, \int_{0}^{T} \int_{0}^{l} H(\theta, z) u(\theta, z) d z d \theta\right)+\right. \\
& \left.+\int_{0}^{y} f\left(z, \int_{0}^{T} \int_{0}^{l} H(\theta, \xi) u(\theta, \xi) d \xi d \theta\right) d z\right] d y
\end{aligned}
$$

где

$$
\begin{aligned}
& h(t, x)=Q(t, x)+\int_{0}^{x} F_{1}(t, x, y) \bar{g}(y) d y, \\
& \Phi(t, x, y)=F_{2}(t, x, y)-\bar{F}\left(t_{0}\right) F_{1}(t, x, y) .
\end{aligned}
$$

Для произвольной функции $l(t, x) \in C^{2,2}(\Omega)$ рассматривается следующая норма:

$$
\|l(t, x)\|_{C}=\max \{|l(t, x)|:(t, x) \in \Omega\} .
$$

Tеорема. Пусть

1) выполняются условия условия (10), (13), (15), $\mu\left(t_{0}\right) \neq 0$;

2) $\sigma=\max \{|h(t, x)|:(t, x) \in \Omega\}<\infty$; 
3) $M=\max \left\{\left|\int_{0}^{x} \Phi(t, x, y)\left[f(y, \gamma)+\int_{0}^{y} f(z, \gamma) d z\right] d y\right|:(t, x) \in \Omega\right\}<\infty$;

4) $\left|f\left(x, \gamma_{1}\right)-f\left(x, \gamma_{2}\right)\right| \leqslant L(x)\left|\gamma_{1}-\gamma_{2}\right|, \delta_{1}=|\gamma| \leqslant \int_{0}^{T} \int_{0}^{l}|H(t, x)| d x d t<\infty$;

5) $\delta_{2}=\max \left\{\int_{0}^{x}|\Phi(t, x, y)|\left[L(y)+\int_{0}^{y} L(z) d z\right] d y:(t, x) \in \Omega\right\}<\infty$, $\rho=\delta_{1} \delta_{2}<1$.

Тогда существует единственная пара решений $\left\{u(t, x) \in C^{2,2}(\Omega), \beta(x) \in\right.$ $\left.C\left(\Omega_{l}\right)\right\}$ обратной задачи (1)-(4).

До ка з а тел в ст в о. Рассмотрим следующий итерационный процесс для уравнения (25):

$$
\begin{aligned}
& u_{0}(t, x)=0 \\
& \begin{aligned}
u_{k+1}(t, x)= & h(t, x)+ \\
+ & +\int_{0}^{x} \Phi(t, x, y)\left[f\left(y, \int_{0}^{T} \int_{0}^{l} H(\theta, z) u_{k}(\theta, z) d z d \theta\right)+\right. \\
& \left.\quad+\int_{0}^{y} f\left(z, \int_{0}^{T} \int_{0}^{l} H(\theta, \xi) u_{k}(\theta, \xi) d \xi d \theta\right) d z\right] d y, \quad k=0,1,2, \ldots
\end{aligned}
\end{aligned}
$$

В силу условий теоремы из (26) получаем следующие оценки:

$$
\left\|u_{1}(t, x)-u_{0}(t, x)\right\|_{C} \leqslant \sigma+M
$$

$$
\begin{aligned}
& \left\|u_{k+1}(t, x)-u_{k}(t, x)\right\|_{C} \leqslant \\
& \leqslant \delta_{1} \max \left\{\int_{0}^{x}|\Phi(t, x, y)|\left[L(y)+\int_{0}^{y} L(z) d z\right] \times\right. \\
& \left.\quad \times\left\|u_{k}(t, y)-u_{k-1}(t, y)\right\|_{C} d y:(t, x) \in \Omega\right\} \leqslant \\
& \quad \leqslant \rho\left\|u_{k}(t, x)-u_{k-1}(t, x)\right\|_{C} .
\end{aligned}
$$

В силу последнего условия теоремы из оценки (28) следует, что оператор в правой части (25) является сжимающим. Из оценок (27) и (28) заключаем, что для оператора (25) существует единственная неподвижная точка (см., например, [30, стр. 389-401]). Следовательно, в области $\Omega$ интегральное уравнение (25) имеет единственное решение. Кроме того, справедлива оценка скорости сходимости

$$
\left\|u_{k+1}(t, x)-u(t, x)\right\|_{C} \leqslant \frac{\rho^{k+1}}{1-\rho}(\sigma+M) .
$$

Подставляя решение уравнения (25) в формулу (24), получим интегральное уравнение для однозначного восстановления вторую неизвестную функцию $\beta(x)$ :

$$
\beta(x)+\int_{0}^{x} \beta(y) d y=\alpha(x),
$$


где

$$
\begin{aligned}
\alpha(x)=\bar{g}(x)-\bar{F}\left(t_{0}\right)\left[f \left(x, \int_{0}^{T}\right.\right. & \left.\int_{0}^{l} H(\theta, z) u(\theta, z) d z d \theta\right)+ \\
& \left.+\int_{0}^{x} f\left(y, \int_{0}^{T} \int_{0}^{l} H(\theta, z) u(\theta, z) d z d \theta\right) d y\right] .
\end{aligned}
$$

Поскольку $\alpha(x) \in C\left(\Omega_{l}\right)$, интегральное уравнение (29) имеет единственное решение на отрезке $\Omega_{l}$.

\section{ORCID}

Турсун Камалдинович Юлдашев: http://orcid.org/0000-0002-9346-5362

\section{БИБЛИОГРАФИЧЕСКИЙ СПИСОК}

1. Алгазин С. Д., Кийко И. А. Флаттер пластин и оболочек. М.: Наука, 2006. 248 с.

2. Абзалимов Р. Р., Саляхова Е. В. Разностно-аналитический метод вычисления собственных значений для уравнений четвертого порядка с разделенными краевыми условиями // Изв. вузов. Матем., 2008. №11. С. 3-11.

3. Джураев Т. Д., Сопуев А. К теории дифференциалъных уравнений в частных производных четвертого порядка. Ташкент: Фан, 2000. 144 с.

4. Мукминов Ф. Х., Биккулов И. М. О стабилизации нормы решения одной смешанной задачи для параболических уравнений 4-го и 6-го порядков в неограниченной области // Матем. сб., 2004. Т. 195, № 3. С. 115-142. doi: 10.4213/sm810.

5. Никишкин В. А. Об асимптотике решения задачи Дирихле для уравнения четвертого порядка в слое // Ж. вычисл. матем. и матем. физ., 2014. Т. 54, № 8. С. 1249-1255. doi: $10.7868 / \mathrm{S} 0044466914080122$.

6. Смирнов М. М. Модельные уравнения смешанного типа четвертого порядка. Л.: ЛГУ, 1972. $123 \mathrm{c}$.

7. Юлдашев Т. К. О смешанной задаче для нелинейного уравнения в частных производных четвертого порядка с отражающим отклонением // Вестн. Южно-Ур. ун-та. Сер. Матем. Мех. Физ., 2011. № 4. С. 40-48.

8. Юлдашев Т. К. О смешанной задаче для нелинейного дифференциального уравнения, содержащего квадрат гиперболического оператора и нелинейное отражающее отклонение // Вестн. Томск. гос. ун-та. Матем. и мех., 2011. № 2(14). С. 59-69.

9. Юлдашев Т. К. Смешанная задача для нелинейного дифференциального уравнения четвертого порядка с малым параметром при параболическом операторе // Ж. вычисл. матем. и матем. физ., 2011. Т. 51, № 9. С. 1703-1711.

10. Юлдашев Т. K. О смешанной задаче для одного нелинейного интегродифференциального уравнения в частных производных четвертого порядка// Журнал СВМО, 2012. Т. 14, №2. С. 137-142.

11. Денисов А. М. Введение в теорию обратных задач. М.: МГУ, 1994. 285 с.

12. Денисов А. М. Обратная задача для квазилинейной системы уравнений в частных производных с нелокальным краевым условием // Ж. вычисл. матем. и матем. физ., 2014. T. 54, № 10. C. 1571-1579. doi : 10.7868/S004446691410007X.

13. А. Б. Костин Обратная задача восстановления источника в параболическом уравнении по условию нелокального наблюдения // Матем. сб., 2013. Т. 204, № 10. С. 3-46. doi : 10 . $4213 / \mathrm{sm} 8104$.

14. Лаврентьев М. М., Савельев Л. Я. Линейные операторы и некорректные задачи. М.: Наука, 1991. 331 с.

15. Мегралиев Я. Т. Об одной обратной краевой задаче для эллиптического уравнения второго порядка с интегральным условием первого рода / Тр. ИММ УрО РАН, Т. 19, 2013. C. $226-235$. 
16. Прилепко А. И., Костин А. Б. О некоторых обратных задачах для параболических уравнений с финальным и интегральным наблюдением // Матем. сб., 1992. Т. 183, № 4 . C. $49-68$.

17. Прилепко А. И., Ткаченко Д. С. Свойства решений параболического уравнения и единственность решения обратной задачи об источнике с интегральным переопределением // Ж. вычисл. матем. и матем. физ., 2003. Т. 43, № 4. С. 562-570.

18. Романов В. Г. Обратные задачи для математической физики. М.: Наука, 1984. 264 с.

19. Юлдашев Т. К. Обратная задача для одного нелинейного интегродифференциального уравнения третьего порядка // Вестн. СамГУ. Естественнонаучн. сер., 2013. № 9/1(110). С. 58-66.

20. Юлдашев Т. К. Об обратной задаче для нелинейных интегро-дифференциальных уравнений высшего порядка// Вестник Воронежского государственного университета. Серия: Физика. Математика, 2014. № 1. С. 153-163.

21. Юлдашев Т. К. Об обратной задаче для квазилинейного уравнения в частных производных первого порядка // Вестн. Томск. гос. ун-та. Матем. и мех., 2012. № 2(18). C. $56-62$.

22. Юлдашев Т. К. Об обратной задаче для системы квазилинейных уравнений в частных производных первого порядка // Вестн. Южно-Ур. ун-та. Сер. Матем. Мех. Физ., 2012. №6. C. 35-41.

23. Юлдашев Т. К. Обратная задача для нелинейного уравнения с псевдопараболическим оператором высокого порядка // Вестн. Сам. гос. техн. ун-та. Сер. Физ.-мат. науки, 2012. № 3(28). C. 17-29. doi: 10.14498/vsgtu1041.

24. Юлдашев Т. К. Обратная задача для нелинейного интегро-дифференциального уравнения с гиперболическим оператором высокой степени // Вестн. Южно-Ур. ун-та. Сер. Матем. Мех. Физ., 2013. Т. 5, № 1. С. 69-75.

25. Юлдашев Т. К., Середкина А. И. Обратная задача для квазилинейных интегродифференциальных уравнений в частных производных высокого порядка // Becmн. Сам. гос. техн. ун-та. Сер. Физ.-мат. науки, 2013. №3(32). С. 46-55. doi: 10.14498/ vsgtu1133.

26. Юлдашев Т. К. О разрешимости смешанной задачи для линейного парабологиперболического интегро-дифференциального уравнения Фредгольма // ЖКурнал CBMO, 2013. Т. 15, № 3. С. 158-163.

27. Юлдашев Т. К. Обратная задача для одного интегро-дифференциального уравнения Фредгольма в частных производных третьего порядка // Вестн. Сам. гос. техн. унта. Сер. Физ.-мат. науки, 2014. №1(34). С. 56-65. doi: 10.14498/vsgtu1299.

28. Юлдашев Т. К. Двойная обратная задача для интегро-дифференциального уравнения Фредгольма эллиптического типа // Вестн. Сам. гос. техн. ун-та. Сер. Физ.-мат. науки, 2014. № 2(35). С. 39-49. doi: 10.14498/vsgtu1306.

29. Юлдашев Т. К., Шабадиков К. Х. Обратная задача для гиперболического интегродифференциального уравнения Фредгольма // Таврический вестник информатики и математики, 2014. №1. C. 73-81, http://tvim.info/files/73_81_Yuldashev.pdf.

30. Треногин В. А. Функиионалъный анализ. М.: Наука, 1980. 495 с.

Поступила в редакцию 29/04/2015;

в окончательном варианте - 14/06/2015;

принята в печать - 08/VIII/2015. 
Vestn. Samar. Gos. Techn. Un-ta. Ser. Fiz.-mat. nauki

[J. Samara State Tech. Univ., Ser. Phys. \& Math. Sci.], 2015, vol. 19, no. 4, pp. $736-749$

ISSN: 2310-7081 (online), 1991-8615 (print)

doi: http://dx.doi.org/10.14498/vsgtu1434

MSC: 45B05

\title{
AN INVERSE PROBLEM FOR A NONLINEAR FREDHOLM INTEGRO-DIFFERENTIAL EQUATION OF FOURTH ORDER WITH DEGENERATE KERNEL
}

\author{
T. K. Yuldashev \\ M. F. Reshetnev Siberian State Aerospace University, \\ 31, pr. "Krasnoyarski Rabochiy", Krasnoyarsk, 660014, Russian Federation.
}

\begin{abstract}
We consider the questions of one value solvability of the inverse problem for a nonlinear partial Fredholm type integro-differential equation of the fourth order with degenerate kernel. The method of degenerate kernel is developed for the case of inverse problem for the considering partial Fredholm type integro-differential equation of the fourth order. After denoting the Fredholm type integro-differential equation is reduced to a system of integral equations. By the aid of differentiating the system of integral equations reduced to the system of differential equations. When a certain imposed condition is fulfilled, the system of differential equations is changed to the system of algebraic equations. For the regular values of spectral parameterthe system of algebraic equations is solved by the Kramer metod. Using the given additional condition the nonlinear Volterra type integral equation of second kind with respect to main unknowing function and the nonlinear Volterra special type integral equation of first kind with respect to restore function are obtained. We use the method of successive approximations combined with the method of compressing maps. Further the restore function is defined. This paper developes the theory of Fredholm integro-differential equations with degenerate kernel.
\end{abstract}

Keywords: inverse problem, integro-differential equation, Fredholm type equation, degenerate kernel, system of integral equations, one valued solvability.

doi: http://dx.doi.org/10.14498/vsgtu1434

\section{ORCID}

Tursun K. Yuldashev: http://orcid.org/0000-0002-9346-5362

\section{REFERENCES}

1. Algazin S. D., Kiyko I. A. Flatter plastin i obolochek [Flutter of plates and shells]. Moscow, Nauka, 2006, 248 pp. (In Russian)

(C) 2015 Samara State Technical University.

Please cite this article in press as:

Yuldashev T. K. An inverse problem for a nonlinear Fredholm integro-differential equation of fourth order with degenerate kernel, Vestn. Samar. Gos. Tekhn. Univ., Ser. Fiz.-Mat. Nauki [J. Samara State Tech. Univ., Ser. Phys. \& Math. Sci.], 2015, vol. 19, no. 4, pp. 736-749. doi: 10.14498/vsgtu1434. (In Russian)

Author Details:

Tursun K. Yuldashev (Cand. Phys. \& Math. Sci.; tursunbay@rambler.ru), Associate Professor, Dept. of Higher Mathematics. 
2. Abzalimov R. R., Salyakhova E. V. A difference-analytical method for the computation of eigenvalues of the fourth-order equations with separated boundary conditions, Russian Math. (Iz. VUZ), 2008, vol. 52, no. 11, pp. 1-9. doi: 10.3103/S1066369X08110017.

3. Dzhuraev T. D., Sopuev A. K teorii differentsial'nykh uravnenii v chastnykh proizvodnykh chetvertogo poriadka [Theory of partial differential equations of fourth order]. Tashkent, Fan, 2000, 144 pp. (In Russian)

4. F. Kh. Mukminov, I. M. Bikkulov Stabilization of the norm of the solution of a mixed problem in an unbounded domain for parabolic equations of orders 4 and 6, Sb. Math., 2004, vol. 195, no. 3, pp. 413-440. doi: 10.1070/SM2004v195n03ABEH000810.

5. Nikishkin V. A. On the asymptotics of the solution of the Dirichlet problem for a fourthorder equation in a layer, Comput. Math. Math. Phys., 2014, vol. 54, no. 8, pp. 1214-1220. doi: 10.1134/S0965542514080107.

6. Smirnov M. M. Modelnyye uravneniya smeshannogo tipa chetvertogo poryadka [Mixed type model equation of fourth order]. Leningrad, Leningrad State University Press, 1972 (In Russian).

7. Yuldashev T. K. On a mixed value problem for nonlinear partial differential equation of the fourth order with reflecting deviation, Vestn. Yuzhno-Ural. Gos. Un-ta. Ser. Matem. Mekh. Fiz., 2011, no. 4, pp. 40-48 (In Russian).

8. Yuldashev T. K. On a mixed value problem for a nonlinear partial differential equation containing a squared hyperbolic operator and nonlinear reflecting deviation, Vestn. Tomsk. Gos. Univ. Mat. Mekh., 2011, no. 2(14), pp. 59-69 (In Russian).

9. Yuldashev T. K. Mixed value problem for a nonlinear differential equation of fourth order with small parameter on the parabolic operator, Comput. Math. Math. Phys., 2011, vol. 51, no. 9, pp. 1596-1604. doi : 10.1134/S0965542511090181.

10. Yuldashev T. K. On a mixed value problem for one nonlinear partial integro-differential equation of the fourth order, Zhurnal SVMO, 2012, vol. 14, no. 2, pp. 137-142 (In Russian).

11. Denisov A. M. Vvedeniye v teoriyu obratnykh zadach [Introduction to the theory of inverse problem]. Moscow, Lomonosov Moscow State University Press, 1994, 285 pp. (In Russian)

12. Denisov A. M. Inverse problem for a quasilinear system of partial differential equations with a nonlocal boundary condition, Comput. Math. Math. Phys., 2014, vol. 54, no. 10, pp. 15131521. doi: 10.1134/S0965542514100066.

13. Kostin A. B. The inverse problem of recovering the source in a parabolic equation under a condition of nonlocal observation, Sb. Math., 2013, vol. 204, no. 10, pp. 1391-1434. doi : 10. 1070/SM2013v204n10ABEH004344.

14. Lavrent'ev M. M., Savel'ev L. Ya. Linear operators and ill-posed problems. New York, Consultants Bureau, 1995, xiv+382 pp.

15. Megraliev Ya. T. On an inverse boundary value problem for a second-order elliptic equation with integral condition of the first kind, Trudy Inst. Mat. i Mekh. UrO RAN, 19, 2013, pp. 226-235 (In Russian).

16. Prilepko A. I., Kostin A. B. On certain inverse problems for parabolic equations with final and integral observation, Russian Acad. Sci. Sb. Math., 1993, vol.75, no. 2, pp. 473-490. doi: 10.1070/SM1993v075n02ABEH003394.

17. Prilepko A. I., Tkachenko D. S. Properties of solutions of a parabolic equation and the uniqueness of the solution of the inverse source problem with integral overdetermination, Comput. Math. Math. Phys., 2003, vol. 43, no. 4, pp. 537-546.

18. Romanov V. G. Inverse Problems of Mathematical Physics. Utrecht, VNU Science Press, 1987, vii+224 pp.

19. Yuldashev T. K. Inverse problem for a nonlinear integral and differential equation of the third order, Vestnik SamGU. Estestvenno-Nauchnaya Ser., 2013, no.9/1(110), pp. 58-66 (In Russian).

20. Yuldashev T. K. On inverse problem for nonlinear integro-differential equations of the higher order, Vestnik Voronezhskogo gosudarstvennogo universiteta. Seriia: Fizika. Matematika [Proceedings of Voronezh State University. Series: Physics. Mathematics], 2014, no. 1, pp. 153-163 (In Russian). 
21. Yuldashev T. K. On the inverse problem for the quasilinear partial differential equation of the first order, Vestn. Tomsk. Gos. Univ. Mat. Mekh., 2012, no.2(18), pp. 56-62 (In Russian).

22. Yuldashev T. K. On an inverse problem for a system of quazilinear equations in partial derivatives of the first order, Vestn. Yuzhno-Ural. Gos. Un-ta. Ser. Matem. Mekh. Fiz., 2012, no. 6, pp. 35-41 (In Russian).

23. Yuldashev T. K. Inverse problem for nonlinear partial differential equation with high order pseudoparabolic operator, Vestn. Samar. Gos. Tekhn. Univ. Ser. Fiz.-Mat. Nauki [J. Samara State Tech. Univ., Ser. Phys. \& Math. Sci.], 2012, no. 3(28), pp. 17-29 (In Russian). doi : 10. 14498/vsgtu1041.

24. Yuldashev T. K. Inverse problem for nonlinear integral differential equation with hyperbolic operator of a high degree, Vestn. Yuzhno-Ural. Gos. Un-ta. Ser. Matem. Mekh. Fiz., 2013, vol. 5, no. 1, pp. 69-75 (In Russian).

25. Yuldashev T. K., Seredkina A. I. Inverse problem for quazilinear partial integro-differential equations of higher order, Vestn. Samar. Gos. Tekhn. Univ. Ser. Fiz.-Mat. Nauki [J. Samara State Tech. Univ., Ser. Phys. \& Math. Sci.], 2013, vol. 3(32), pp. 46-55 (In Russian). doi : 10. 14498/vsgtu1133.

26. Yuldashev T. K. On solvability of mixed value problem for linear parabolo-hyperbolic Fredholm integro-differential equation, Zhurnal SVMO, 2013, vol. 15, no.3, pp. 158-163 (In Russian).

27. Yuldashev T. K. Inverse problem for a Fredholm third order partial integro-differential equation, Vestn. Samar. Gos. Tekhn. Univ. Ser. Fiz.-Mat. Nauki [J. Samara State Tech. Univ., Ser. Phys. \& Math. Sci.], 2014, no.1(34), pp. 56-65 (In Russian). doi: 10.14498/ vsgtu1299.

28. Yuldashev T. K. A double inverse problem for Fredholm integro-differential equation of elliptic type, Vestn. Samar. Gos. Tekhn. Univ. Ser. Fiz.-Mat. Nauki [J. Samara State Tech. Univ., Ser. Phys. \& Math. Sci.], 2014, no. 2(35), pp. 39-49 (In Russian). doi: 10.14498/ vsgtu1306.

29. Yuldashev T. K., Shabadikov K. X. The inverse problem for the hyperbolic Fredholm integro-differential equations, Tavricheskii vestnik informatiki i matematiki [Tourida journal of computer science theory and mathematics], 2014, no. 1, pp. 73-81 (In Russian), http: //tvim.info/files/73_81_Yuldashev.pdf.

30. Trenogin V. A. Funktsional'nyi analiz [Functional analyses]. Moscow, Nauka, 1980, 495 pp. (In Russian)

Received 29/04/2015;

received in revised form 14/06/2015;

accepted 08/VIII/2015. 